\title{
Hernia umbilical en pacientes cirróticos con ascitis. ¿Tratamiento conservador o quirúrgico?
}

\author{
Doyler Cubas-García ${ }^{1}$, José Gálvez-Olortegui ${ }^{2}$, José Caballero-Alvarado ${ }^{3,4}$, \\ Pedro Delgado-Guillena ${ }^{5,6}$ y Edward Chávez-Cruzado ${ }^{2,4}$
}

\section{Umbilical hernia in liver cirrhosis with ascites. Conservative or Surgical treatment?}

Ascites is the most common complication in patients with liver cirrhosis. Umbilical hernias (HU) occur in $20 \%$ of these patients and $40 \%$ in those with severe ascites. HU occurs due to increased intra-abdominal pressure, weakening of the abdominal fascia and loss of muscle mass. In addition, they have a tendency to enlarge rapidly and present high risk of complications that threaten the patient's life. The treatment of the uncomplicated HU is controversial, both the surgical management (herniorrhaphy) and the conservative management (control of ascites) present high rate of complications, consequently high morbidity and mortality. Currently, umbilical herniorrhaphy is recommended with prior control of ascites in uncomplicated HU management, it reduces the risk of surgical wound infection, evisceration, ascites drainage, peritonitis, and it reduces up to $41 \%$ of HU recurrence. The success of this approach also depends on the degree of liver dysfunction. The treatment of complicated HU is surgical (herniorrhaphy without mesh), with lower mortality rate compared to conservative management. Studies reveal advantages of umbilical herniorrhaphy laparoscopy (minimally invasive and stress-free) compared to open surgery; however there is still no evidence about it.

Key words: Umbilical hernia; Herniorrhaphy; Ascites; Hepatic cirrhosis.

\section{Resumen}

La ascitis es la complicación más común en los pacientes con cirrosis hepática. Las hernias umbilicales (HU) ocurren en $20 \%$ de estos pacientes y $40 \%$ en aquellos con ascitis severa. La HU ocurre debido al aumento de presión intraabdominal, al debilitamiento de la fascia abdominal y la pérdida de masa muscular. Además, tienen tendencia a aumentar rápidamente y presentar alto riesgo de complicaciones que amenazan la vida del paciente. El tratamiento de la HU no complicada es controversial, tanto el manejo quirúrgico (herniorrafia) como el manejo conservador (control de ascitis) presentan alta tasa de complicaciones, en consecuencia incrementa la morbimortalidad. Actualmente, se recomienda herniorrafia umbilical con previo control de la ascitis en el manejo de HU no complicada, reduce el riesgo de infección de herida operatoria, evisceración, drenaje de ascitis, peritonitis y reduce hasta $41 \%$ la recidiva de HU. El éxito de este enfoque también depende del grado de disfunción hepática. El tratamiento de la HU complicada es quirúrgico (herniorrafia sin malla), con menor tasa de mortalidad respecto al manejo conservador. Estudios revelan ventajas de la herniorrafia umbilical laparoscópica (mínimamente invasiva y sin tensión) en comparación a la cirugía abierta, sin embargo, aún no hay evidencia al respecto.

Palabras clave: Hernia umbilical; Herniorrafia; Ascitis; Cirrosis hepática.

\section{Introducción}

La cirrosis hepática es el estadio final de enfermedades hepáticas progresivas crónicas, caracterizado por el desarrollo de fibrosis tisular difusa y la sustitución de la arquitectura normal por nódulos de regeneración. Con el progreso de la enfermedad aparecen complicaciones, donde la ascitis es la más frecuente'.

En los pacientes cirróticos, la ascitis ejerce un aumento de la presión intraabdominal, que junto al debilitamiento de la fascia abdominal, la pérdida
Facultad de Medicina

Universidad Nacional de Trujillo, Trujillo, Perú. ¿Unidad Generadora de Evidencias y Vigilancia Epidemiológica, Scientia Clinical and

Epidemiological Research Institute, Trujillo, Peru ${ }^{3}$ Servicio de Cirugia, Hospital Regional Docente de Trujillo, Trujillo, Perú.

4Escuela de Postgrado Universidad Privada Anterior Orrego, Trujillo, Perú.

${ }^{5}$ Unidad de Investigación Clínica, Scientia Clinical and Epidemiological Research Institute, Trujillo, Perú. ${ }^{6}$ Servicio de Aparato Digestivo, Hospital General de Granollers, Barcelona, España.

Recibido el 14 de febrero 2017, aceptado el 1 de agosto de 2017.

Correspondencia a: Edward Chávez-Cruzado edwardchavezc@hotmail.com 
de masa muscular y la presencia de dilatación de la vena umbilical secundaria a la hipertensión portal; constituyen condiciones que aperturan el anillo fibroso obturado en la cicatriz umbilical, protruyendo el peritoneo, lo que da inicio a la formación de hernias umbilicales (HU ${ }^{2,3}$. Adicionalmente, existen otros factores de riesgo relacionados con la génesis de HU en los pacientes cirróticos, como hiponatremia, fosfatasa alcalina sérica baja y score Child-Pugh alto ${ }^{4}$.

Las HU se presentan en el $2 \%$ de la población general, en pacientes cirróticos compensados en el $20 \%$ y en pacientes cirróticos con ascitis severa esta cifra se eleva hasta el $40 \%$. Estas HU presentan tendencia a aumentar rápidamente y originar mayor riesgo de complicaciones que amenazan la vida del paciente, debido a la gran presión intraabdominal ${ }^{2,5}$, situaciones en las que es necesario realizar un manejo de urgencia.

El tratamiento de elección de $\mathrm{HU}$, en pacientes sin comorbilidades, es quirúrgico y presenta baja tasa de complicaciones; sin embargo, en los pacientes cirróticos con ascitis, la tasa es alta y tiene un elevado riesgo de morbimortalidad perioperatoria que se incrementa progresivamente en relación con la gravedad de la disfunción hepática preoperatoria ${ }^{6}$. Si bien es cierto, en HU complicadas (atascamiento y/o estrangulamiento) en pacientes con ascitis, el tratamiento debe ser quirúrgico, teniendo como objetivo principal resolver la complicación; existe controversia respecto al tratamiento ideal (quirúrgico o conservador) en los pacientes cirróticos con ascitis y HU no complicada (Tabla 1).

\section{Manejo quirúrgico de HU no complicada}

Consiste en herniorrafia umbilical con malla de manera electiva, es seguro, eficaz y reduce la recurrencia de hernia, además ha demostrado ser superior a la herniorrafia sola en las reparaciones electivas. Aunque hay autores que refieren que existe un mayor riesgo de infección de sitio operatorio y fuga de líquido a través de la malla con necesidad de removerla, no hay evidencia registrada que avale esta afirmación ${ }^{2,7-9}$. La elección de las técnicas de colocación de malla tanto onlay, inlay, sublay $y$ underlay en la cirugía abierta es controvertida, ya que se acompaña cada una de complicaciones específicas, es por eso que el acceso laparoscópico, procedimiento mínimamente invasivo y sin tensión, ha sido una propuesta para su tratamiento, en el que se coloca la malla por vía intraperitoneal y se fija en la pared abdominal para cerrar la entrada de la her- nia; a pesar que no hay estudios aleatorizados se han descrito ciertas ventajas respecto a la cirugía abierta tales como disminución del dolor, reducción de pérdida de sangre, recuperación temprana, mínima fuga de ascitis a través de la herida, menor pérdida de electrolitos y proteínas por la no exposición de las vísceras, recuperación temprana y reducción de recurrencia de la $\mathrm{HU}^{2}$. Sin embargo, en pacientes descompensados el tratamiento quirúrgico se asocia a altas tasas de complicaciones perioperatorias, que incluyen infección de sitio operatorio, dehiscencia, fístula ascítica, insuficiencia hepática y peritoni$\operatorname{tis}^{2,5,6}$.

\section{Tratamiento conservador de hernia umbilical (HU) no complicada}

Se basa en el control de ascitis mediante el uso de 2 diuréticos (espironolactona y furosemida en una proporción 100:40 mg por día, incrementando progresivamente en caso sea necesario hasta 400 y 160 mg respectivamente), soporte nutricional, albúmina intravenosa con objetivo de aumentar la albúmina sérica a más de $3 \mathrm{~g} / \mathrm{dL}^{9,10}$, restricción de sodio a 88 $\mathrm{mEq}(2.000 \mathrm{mg})$ por día, y finalmente mediante el uso de paracentesis evacuadora periódica ${ }^{2}$. Este tratamiento reduciría las potenciales complicaciones de HU o de una futura intervención quirúrgica; aun así, existen riesgos asociados como incarceración herniaria, evisceración, fuga de líquido ascítico, ulceración, ruptura, necrosis de la piel que lo recubre $\mathrm{y}$ alto riesgo de peritonitis bacteriana ${ }^{2,9}$.

\section{Tratamiento de hernia umbilical (HU) complicada}

La morbilidad en pacientes cirróticos, con ascitis y hernia umbilical complicada es elevada, pudiendo ser la postoperatoria de hasta $71 \%$; mientras que el riesgo de mortalidad puede variar entre $60-80 \%$ tras el tratamiento conservador y de 6-20\% tras la reparación quirúrgica de urgencia ${ }^{2,8}$. En base a esto, se puede plantear que en pacientes cirróticos con ascitis y HU complicada, el manejo debe realizarse con urgencia ${ }^{2,3}$. La resolución de la patología herniaria complicada en estos pacientes en caso sea posible, debe realizarse sin prótesis (para disminuir el riesgo de infección) e idealmente con la técnica de Mayo (con o sin resección de ombligo) con sutura secundaria de flap (con sutura irreabsorbible, preferentemente polipropileno 0 y 2-0). Esta técnica es más anatómica, hermética, segura y evita las complicaciones de la prótesis, sobre todo en estos pacientes. 


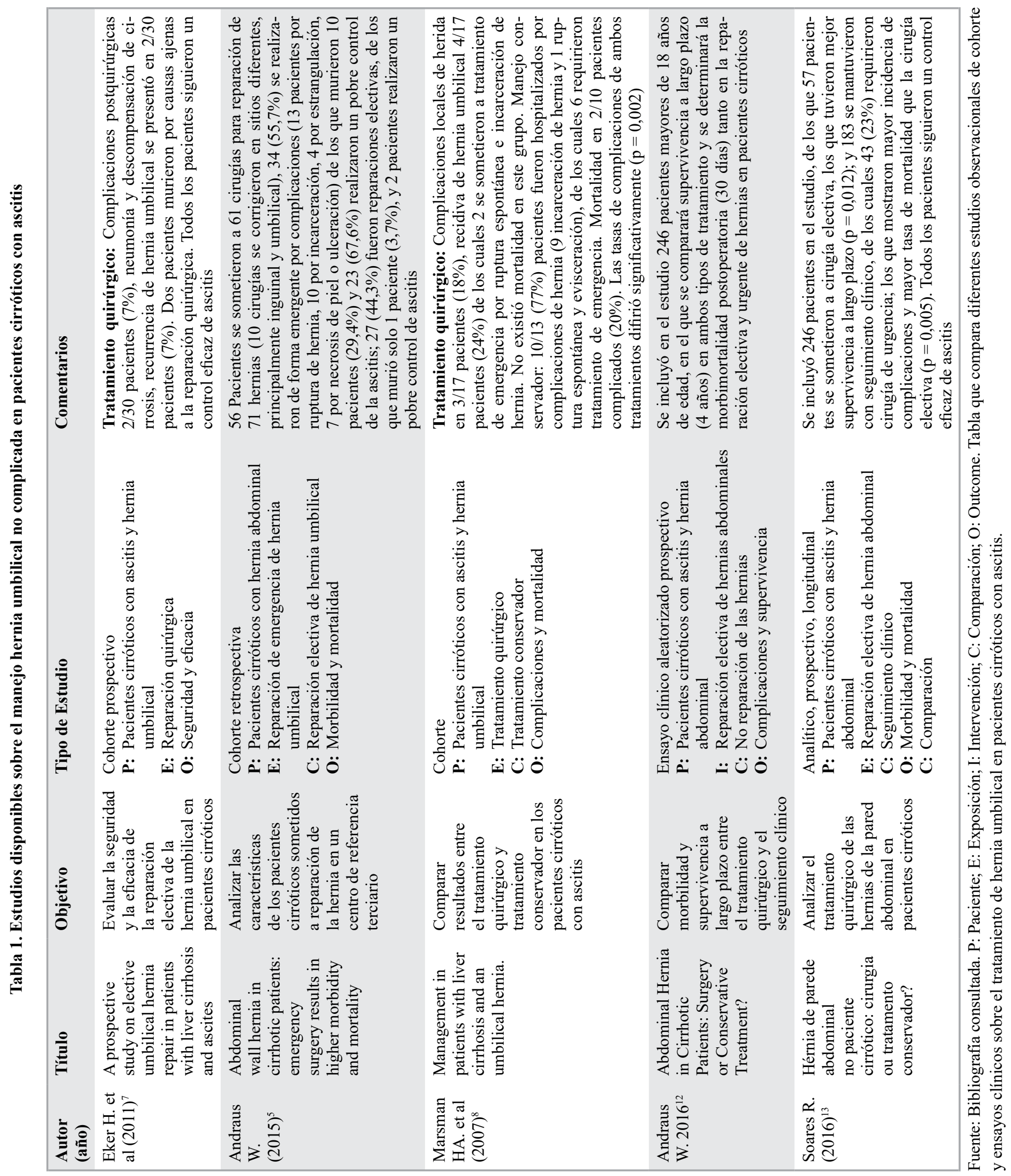


Tratamiento mixto (médico y conservador) de la hernia umbilical (HU) no complicada

Autores recomiendan la reparación quirúrgica de la hernia umbilical con control eficaz de la ascitis, o durante el trasplante hepático cuando el control de la ascitis falla ${ }^{2}$. El enfoque es prometedor, ya que el control eficaz de la ascitis reduce el riesgo de infección de herida operatoria, evisceración, drenaje de la ascitis, peritonitis y reduce hasta en $41 \%$ de recidiva de la hernia umbilical. Asimismo se señala que el éxito quirúrgico depende también del grado de disfunción hepática ${ }^{2,4}$, reportando asociación entre score MELD (del inglés Model for End-stage Liver Disease) mayor de 15 y el Grado C del Score Child-Pugh con el aumento de la tasa de morbimortalidad $^{5,11,12}$; considerando además a la presencia de várices esofágicas, la edad mayor a 65 años, el nivel de albúmina inferior a 3,0 g/dl y la hiponatremia, como predictores adversos ${ }^{2,4}$.

\section{Cuidados perioperatorios en el manejo de hernia umbilical (HU)}

Previamente al tratamiento quirúrgico, los pacientes deben estar sujetos a una resucitación adecuada de fluidos (cristaloides con solución de albúmina), se sugiere hacer la reparación bajo anestesia local (lidocaina 1\%) y sedación intravenosa (midazolan o fentanilo) o anestesia regional (contraindicado en coagulopatía severa y/o trombocitopenia por riesgo de hematoma epidural espinal) en casos de apertura herniaria pequeña, antibióticos (cefalosporinas de primera o segunda generación) para prevenir o tratar la infección de líquido ascítico; incluso se sugiere limpiar y proteger la piel con un apósito estéril en casos de ruptura o ulceración cutánea de la $\mathrm{HU}$, a fin de disminuir el riesgo de sobreinfección de ascitis ${ }^{2,13,14}$. Respecto a los cuidados postoperatorios, autores consideran mantener fluidoterapia durante 24 a 48 h, con mantenimiento del tratamiento médico de la ascitis para limitar episodios posteriores, cultivo de líquido ascítico o drenajes en caso sea necesario en el manejo de antibioticoterapia (HU complicadas), la incisión de la piel debe ser inspeccionada diariamente para detectar fugas de líquido ascítico o recidivas tempranas, el drenaje no debe ser removido antes de una semana de acuerdo al estado de la herida y las suturas deben mantenerse durante al menos dos semanas ${ }^{13}$.

Los estudios publicados que comparan el tratamiento conservador y quirúrgico de $\mathrm{HU}$ en pacientes cirróticos con ascitis, son observacionales y han evaluado diversos desenlaces como morbilidad, mortalidad, supervivencia, etc. Sin embargo un ensayo clínico aleatorizado ha finalizado en el 2016 $(\text { Tabla } 1)^{15}$. Este estudio realizó seguimiento a 246 pacientes por 5 años, y su reporte permitirá comparar la mortalidad y supervivencia a largo plazo en ambos tipos de tratamiento.

\section{Conclusión}

Aunque no existe suficiente evidencia, actualmente el tratamiento quirúrgico de la hernia umbilical previo control eficaz de la ascitis, es el más recomendado en esta población específica, ya que el tratamiento quirúrgico o conservador realizado aisladamente se relaciona con alta morbimortalidad.

\section{Responsabilidades éticas}

Protección de personas y animales. Los autores declaran que para esta investigación no se han realizado experimentos en seres humanos ni en animales.

Confidencialidad de los datos. Los autores declaran que en este artículo no aparecen datos de pacientes.

Derecho a la privacidad y consentimiento informado. Los autores declaran que en este artículo no aparecen datos de pacientes.

\section{Financiación}

Los autores declaran no haber recibido ninguna financiación para la realizacion de este trabajo.

\section{Conflicto de intereses}

Los autores declaran no tener ningún conflicto de intereses. 


\section{Bibliografía}

1. Buey LG, Mateos FG. Cirrosis hepatica. Medicine (Baltimore). 2012;11:625-33.

2. Coelho JCU, Claus CMP, Campos ACL, Costa MAR, Blum C. Umbilical hernia in patients with liver cirrhosis: A surgical challenge. World J Gastrointest Surg. 2016;8:476-82.

3. Telem DA, Schiano T, Divino CM. Complicated hernia presentation in patients with advanced cirrhosis and refractory ascites: Management and outcome. Surgery. 2010;148: 538-43.

4. Wang R, Qi X, Peng Y, Deng H, Li J, Ning $Z$, et al. Association of umbilical hernia with volume of ascites in liver cirrhosis: a retrospective observational study. J Evid Based Med. 2016;9: 170-80.

5. Andraus W, Pinheiro RS, Lai Q, Haddad LB, Nacif LS, D'Albuquerque LA, et al. Abdominal wall hernia in cirrhotic patients: emergency surgery results in higher morbidity and mortality. BMC Surg. 2015;15:65.

6. Choi SB, Hong KD, Lee JS, Han HJ,
Kim WB, Song TJ, et al. Management of umbilical hernia complicated with liver cirrhosis: An advocate of early and elective herniorrhaphy. Dig Liver Dis. 2011;43:991-5.

7. Triantos CK, Nikolopoulou V, Kehagias I, Burroughs AK. Surgical Repair of Umbilical Hernias in Cirrhosis With Ascites. Am J Med Sci. 2011;341:222-6.

8. Chatzizacharias NA, Bradley JA, Harper S, Butler A, Jah A, Huguet E, et al. Successful surgical management of ruptured umbilical hernias in cirrhotic patients. World J Gastroenterol. 2015;21:3109-13.

9. Eker HH, Van Ramshorst GH, De Goede B, Tilanus HW, Metselaar HJ, De Man RA, et al. A prospective study on elective umbilical hernia repair in patients with liver cirrhosis and ascites. Surgery. 2011;150:542-6.

10. Marsman HA, Heisterkamp J, Halm JA, Tilanus HW, Metselaar HJ, Kazemier G. Management in patients with liver cirrhosis and an umbilical hernia. Surgery. 2007;142:372-5.

11. Ecker BL, Bartlett EK, Hoffman RL,
Karakousis GC, Roses RE, Morris JB, et al. Hernia repair in the presence of ascites. J Surg Res. 2014;190:471-7.

12. Espinoza G R. Accordion: sistema de clasificación de gravedad de las complicaciones quirurgicas. Rev Chil Cir 2010;62:314-5

13. Dokmak S, Aussilhou B, Belghiti J. Umbilical hernias and cirrhose. J Vise Surg. 2012;149:e32-9.

14. Rodríguez JA, Hinder RA. Surgical management of umbilical hernia. Oper Tech Gen Surg. 2004;6:156-64.

15. Andraus W. Abdominal Hernia in Cirrhotic Patients: Surgery or Conservative Treatment? - Tabular view - ClinicalTrials.gov [Internet], [cited 2017 Jan 18]. Available from: https://clinicaltrials.gov/ct2/show/study/ NCT02787772

16. Pinheiro RSN. Hernia de parede abdominal no paciente cirrotico: cirurgia ou tratamento conservador? [Internet]. Biblioteca Digital de Teses e Dissertacoes da Universidade de Sao Paulo; 2016 [cited 2017 Jan 30]. Available from: http://www. teses.usp.br/teses/disponiveis/5/5168/tde20092016-162922/ 